\title{
Avaliação da Aprendizagem em Gerenciamento de Custos de Materiais Aplicado à Enfermagem Hospitalar
}

\author{
Marta Cristiane Alves Pereira*, Igor Simões da Silva Isaac, Josana Camilo Bodnar, Jéssica \\ Amici Moraes, Geovana Cristina Teso da Silva, Rosângela Andrade Aukar de Camargo \\ Escola de Enfermagem de Ribeirão Preto da Universidade de São Paulo
}

*Autora para correspondência: martacris@eerp.usp.br

\section{RESUMO}

Pesquisa exploratório-descritiva com objetivo de descrever as contribuições da WebQuest sobre gerenciamento de custos aplicados à Enfermagem, na perspectiva dos estudantes de enfermagem. Participaram 25 estudantes do curso de graduação em Enfermagem, mediante aprovação pelo Comitê de Ética em Pesquisa. A plataforma Moodle foi utilizada para disponibilização da WebQuest. Na avaliação da aprendizagem, foi identificada a predominância de acertos e não foi constatada diferença estatisticamente significante entre as notas dos questionários aplicados antes e depois da aula. Por outro lado, observou-se diferença na redução do tempo utilizado para se responder ao questionário. $\mathrm{Na}$ avaliação da proposta, considerando-se a autoavaliação e a avaliação do grupo, foi atribuída nota média de 96,34, com predominância de desempenho excelente e satisfatório, no atendimento aos requisitos estabelecidos. Os estudantes reconheceram a importância da temática na formação do enfermeiro; manifestaram satisfação na utilização de estratégias ativas e colaborativas para simulação da realidade, utilizando tecnologias da informática e informação; apresentaram sugestões relacionadas ao tempo disponibilizado para as atividades em sala de aula. Os resultados evidenciam o potencial de aprendizagem proporcionado pela WebQuest como estratégia de ensino crítica e reflexiva, que contribui para o desenvolvimento de competências inerentes ao processo de tomada de decisão, enquanto fundamento para o gerenciamento de custos de materiais em Enfermagem.

Palavras-Chave: Enfermagem; Tecnologia Educacional; Gerenciamento.

\begin{abstract}
This exploratory and descriptive research aims to describe the contributions of the WebQuest on Cost Management applied to Nursing, from the perspective of nursing students. Sample consisted of 25 students of Undergraduate Nursing, under approvement of the Research Ethics Committee. Moodle platform was employed in order to provide the WebQuest. In the assessment of learning was identified predominance of correct answers, was not found statistically significant differences between the scores of questionnaires before and after class, but significant differences were observed in reducing the time taken to answer the questionnaire. In suggestion evaluation, selfassessment and group assessment was assigned mean score of 96.34, most excellent and satisfactory performance in meeting the requirements determined. The students identified the importance of the subject for the nurse's development; showed satisfaction by using active and collaborative strategies for simulating reality, by the use of information technology and informatics; showed suggestions related to the time invested on the assignments in class. The results show the potential of learning proved by WebQuest like critical and reflective teaching strategy, that contributes to the development of inherent competencies to the process of making decision, while groundwork to costs management with nursing materials.
\end{abstract}

Keywords: Nursing; Education Technology; Management.

\section{Introdução}

As tecnologias da informação e comunicação permitem desenvolver práticas de aprendizagem que podem ser consideradas alicerces de novas relações com o saber, pois os papéis tradicionais de professores e alunos sofrem mudanças profundas, baseadas no diálogo, na construção colaborativa do conhecimento, na provocação à autoria criativa do aprendiz, ao invés da mera transmissão de saberes (SILVA \& GLARO, 2007; LIMA \& NASCIMENTO, 2013).

O desenvolvimento de competências profissionais requer oportunidades de aprendizagem que 
favorecem a reflexão crítica, estimulam a interação e a criatividade e centram-se na avaliação e autoavaliação da aprendizagem, em situações que envolvem a resolução de problemas concretos, vivenciados no cotidiano do trabalho do enfermeiro, passíveis de serem alcançados por meio de recursos tecnológicos simples e acessíveis (PEREIRA et al., 2010; PEREIRA et al., 2014; TEIXEIRA et al., 2015).

Nessa perspectiva, por meio da metodologia WebQuest, é possível despertar o desenvolvimento de atividades interativas, colaborativas e dinâmicas que estimulam autonomia e senso crítico, bem como a responsabilidade pelos resultados alcançados no processo de aprendizagem (PEREIRA et al., 2010; ZHENG et al., 2008; SANDORD et al., 2010; TIBES et al., 2017).

Ademais, a WebQuest prevê ambientes autênticos que podem ser usados junto a grupos com diferentes estilos de aprendizagem e em vários momentos da formação, por meio da integração de diversos conteúdos disciplinares (MAXWELL, 2009; PIERCY, 2004; MARUXO et al., 2015).

Diante do exposto, esta pesquisa tem como objetivo: avaliar a contribuição do Ambiente Virtual de Aprendizagem (AVA) para o aprendizado sobre gerenciamento de custos de coberturas para curativos aplicado à enfermagem hospitalar.

\section{Metodologia}

$\mathrm{O}$ estudo foi realizado em uma universidade pública, no interior do estado de São Paulo. Em sua implementação, utilizou-se a infraestrutura do Laboratório de Informática, que possui projetor multimídia e 34 microcomputadores com acesso à internet.

A população foi constituída por alunos do último semestre do curso de bacharelado em Enfermagem, matriculados na disciplina Organização e Gestão em Saúde e Enfermagem na Atenção Hospitalar, totalizando trinta participantes. A amostra foi constituída por aqueles que concordaram em participar das atividades propostas no decorrer do processo de implementação.
Previu-se duração de noventa minutos para a implementação da WebQuest. Inicialmente, apresentaram-se os objetivos pretendidos, as estratégias a serem adotadas, a sequência de atividades programadas e os critérios de avaliação, destacando-se que os exercícios não caracterizariam atividade obrigatória.

O AVA contempla a abordagem de conteúdos nas seguintes temáticas: economia da saúde, gerenciamento de recursos materiais em Enfermagem e tratamento de lesões por pressão ${ }^{1}$. As oportunidades de aprendizagem "simulam situações reais vivenciadas por enfermeiros no contexto dos serviços de saúde, utilizando recursos simples e acessíveis de tecnologias da informática e informação" (PEREIRA et al., 2014, p. 328). Na avaliação da contribuição do AVA para o aprendizado, foram realizadas avaliações somativas e formativas.

$\mathrm{Na}$ avaliação formativa, inerente ao alcance dos objetivos educacionais, utilizaram-se as ferramentas da plataforma Moodle (pasta de arquivos, tarefa, fórum). Na avaliação somativa da aprendizagem, aplicaram-se questionários de associação e de múltipla escolha (Figura 1 e Figura 2), relacionados a conceitos básicos abordados no decorrer da WebQuest e disponibilizados na plataforma Moodle, antes e depois da implementação das atividades pelos alunos.

$\mathrm{Na}$ avaliação dos alunos, utilizou-se um roteiro constituído de duas partes: uma em que eles avaliam a proposta desenvolvida e outra constando a sua autoavaliação e a do grupo de trabalho.

A primeira parte é constituída de perguntas sobre a metodologia utilizada e o ambiente virtual de aprendizagem. Na segunda, o aluno responde a perguntas sobre seu desempenho individual e do grupo responsável pelo cumprimento da tarefa, atribuindo um valor a partir de uma escala constando as variáveis: insatisfatório (25\%), razoável $(50 \%)$, satisfatório $(75 \%)$ e excelente $(100 \%)$. Tal material foi disponibilizado na plataforma Moodle no formato de questionário de associação com questões de múltipla escolha.

Nesse processo, foi aplicada análise estatística utilizando o programa Microsoft Office Excel $2010^{\circledR} \mathrm{e}$ o software $R$. Vale ressaltar que as médias aritméticas 


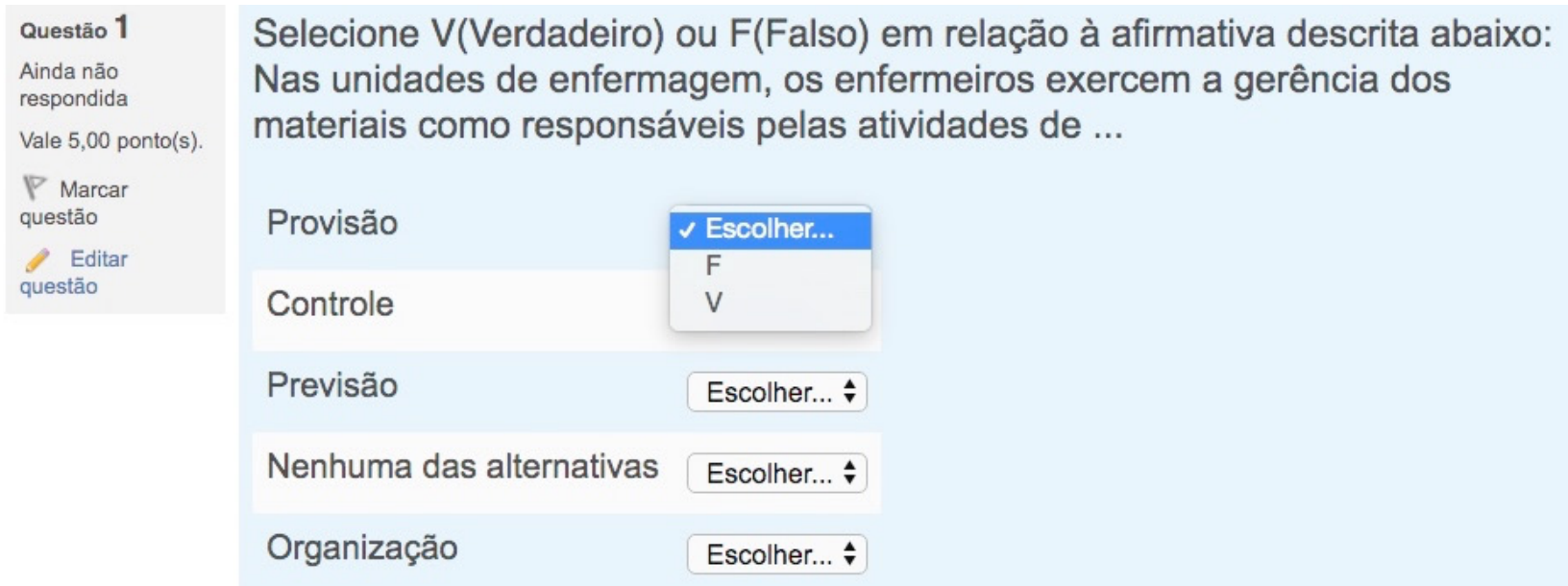

Figura 1 - Questionário para avaliação somativa - Parte 1.

Questão 2
Ainda não
respondida
Vale 5,00 ponto(s).
$P$ Marcar
questão
Editar
questão

\section{Relacione os itens correspondentes descritos nas colunas abaixo:}

O conjunto de ações que os gestores tomam para satisfazer os clientes enquanto, continuamente, reduzem e controlam os custos.

Diz respeito à forma de calcular os custos ou de apropriação dos custos aos produtos e serviços.

Estabelece que o nível de reposição será uma quantidade de material necessário para atender ao período de abastecimento, tendo em vista a expectativa de consumo, mais o estoque de reserva. Sempre que o nível de estoque de um determinado item atingir esse valor, será feito o pedido.

Durante o período de renovação, que é o tempo que decorre entre dois pedidos consecutivos, podem ocorrer algumas falhas, motivadas, por exemplo, por atrasos por parte dos fornecedores na entrega dos produtos ou por aumento de demanda. Para evitar falta de produtos e compras emergenciais, introduz-se este conceito de estoque, que é uma quantidade de material para suprir eventuais necessidades do sistema.

Destina-se à escolha da melhor estratégia para atingir um determinado objetivo, ou seja, trata-se de estudo comparativo de alternativas para executar uma mesma ação.

Escolher...

Figura 2 - Questionário para avaliação somativa - Parte 2. 
e respectivos desvios padrão dos questionários em que foi atribuída uma nota são disponibilizados automaticamente pela plataforma Moodle.

O projeto foi aprovado junto ao Comitê de Ética em Pesquisa da Escola de Enfermagem de Ribeirão Preto-USP (Protocolo 278.705/2013), com dispensa da obtenção do Termo de Consentimento Livre e Esclarecido TCLE junto aos estudantes.

\section{Resultados}

Participaram do estudo 25 alunos, número correspondente a $83,3 \%$, dos graduandos em curso na disciplina Organização e Gestão em Saúde e Enfermagem na Atenção Hospitalar, no último semestre do curso de bacharelado em Enfermagem. Em comparação ao tempo de aula previsto, a dedicação às atividades correspondeu a três horas aproximadamente.

As tarefas foram apreciadas sequencialmente e de modo interdependente. A avaliação foi pautada nos aspectos relacionados tanto ao processo de tomada de decisão quanto à aquisição de coberturas para curativos (necessidades dos pacientes, recomendações da literatura, especificidades dos referidos produtos, custos e recursos envolvidos) sem deixar de lado a análise da relação custo-benefício a ser apresentada na justificativa, vinculada às funções de previsão e provisão.

$\mathrm{Na}$ análise de itens específicos de cada exercício, os erros ocorreram predominantemente na definição das quantidades das coberturas a serem requisitadas, algo realizado por 17 (68\%) dos estudantes, bem como na programação das quantidades anuais das coberturas para todos os pacientes e na indicação das coberturas para os curativos considerando as necessidades de cada paciente, deslize cometido por $12(48 \%)$ dos estudantes.

Os acertos corresponderam sobretudo à codificação e à descrição das coberturas e foram obtidos por $23(92 \%)$ dos estudantes. Além disso, respostas corretas fizeram-se presentes na definição das quantidades de acordo com as necessidades individuais dos pacientes e na realização da classificação $\mathrm{ABC}$, algo assinalado por 18 (72\%) dos alunos. Ao considerar a totalidade dos exercícios, constatou-se predominância de acertos em comparação aos erros e itens não preenchidos (Tabela 1).

$\mathrm{Na}$ avaliação somativa, foram observados aumento da nota média dos estudantes e redução do tempo médio utilizado para responder o questionário aplicado antes e depois da aula (Tabela 2).

Ao comparar as notas médias entre os 22 estudantes que responderam ao questionário aplicado antes da aula e 13 estudantes depois da aula, não foi constatada diferença estatisticamente significante $(p=0,1813)$. Mas se verificou diferença na redução do tempo utilizado para responder ao questionário ( $\mathrm{p}$ menor que 0,05 ), tal como pode ser observado na Figura 3.

$\mathrm{Na}$ análise das respostas dos estudantes referentes às "perspectivas futuras", eles demonstraram

\begin{tabular}{|c|c|c|c|c|c|c|}
\hline \multirow[b]{2}{*}{ Itens } & \multicolumn{2}{|c|}{ Correto } & \multicolumn{2}{|c|}{ Incorreto } & \multicolumn{2}{|c|}{ Não realizou } \\
\hline & $\mathrm{n}$ & $\%$ & $\mathrm{n}$ & $\%$ & $\mathrm{~N}$ & $\%$ \\
\hline \multicolumn{7}{|l|}{ Exercício 1} \\
\hline Indicação da cobertura & 13 & 52 & 12 & 48 & 0 & 0 \\
\hline Quantidade semanal & 18 & 72 & 7 & 28 & 0 & 0 \\
\hline Justificativa & 17 & 68 & 6 & 24 & 2 & 8 \\
\hline \multicolumn{7}{|l|}{ Exercício 2} \\
\hline Descrição das coberturas & 23 & 92 & 2 & 8 & 0 & 0 \\
\hline
\end{tabular}




\begin{tabular}{|c|c|c|c|c|c|c|}
\hline Quantidade anual & 13 & 52 & 12 & 48 & 0 & 0 \\
\hline Classificação $A B C$ & 18 & 72 & 5 & 20 & 2 & 8 \\
\hline \multicolumn{7}{|l|}{ Exercício 3} \\
\hline Codificação das coberturas & 23 & 92 & 2 & 8 & 0 & 0 \\
\hline Especificação das coberturas & 13 & 52 & 12 & 48 & 0 & 0 \\
\hline Quantidade requisitada & 8 & 32 & 17 & 68 & 0 & 0 \\
\hline
\end{tabular}

Tabela 1 - Percentual de itens com respostas corretas dos exercícios realizados pelos estudantes de graduação em Enfermagem ( $\mathrm{n}=25)$. Ribeirão Preto-SP, 2013.

\begin{tabular}{|c|c|c|c|c|}
\hline Questionário & Nota Questão 1 & Nota Questão 2 & Nota Total & Tempo (min) \\
\hline Antes & 4,73 & 2,38 & 7,11 & $09: 59$ \\
\hline Depois & 5,00 & 3,65 & 8,65 & $02: 41$ \\
\hline
\end{tabular}

Tabela 2 - Distribuição das notas e tempos médios dos estudantes ao responder o questionário antes e depois da aula. Ribeirão Preto-SP, 2013.
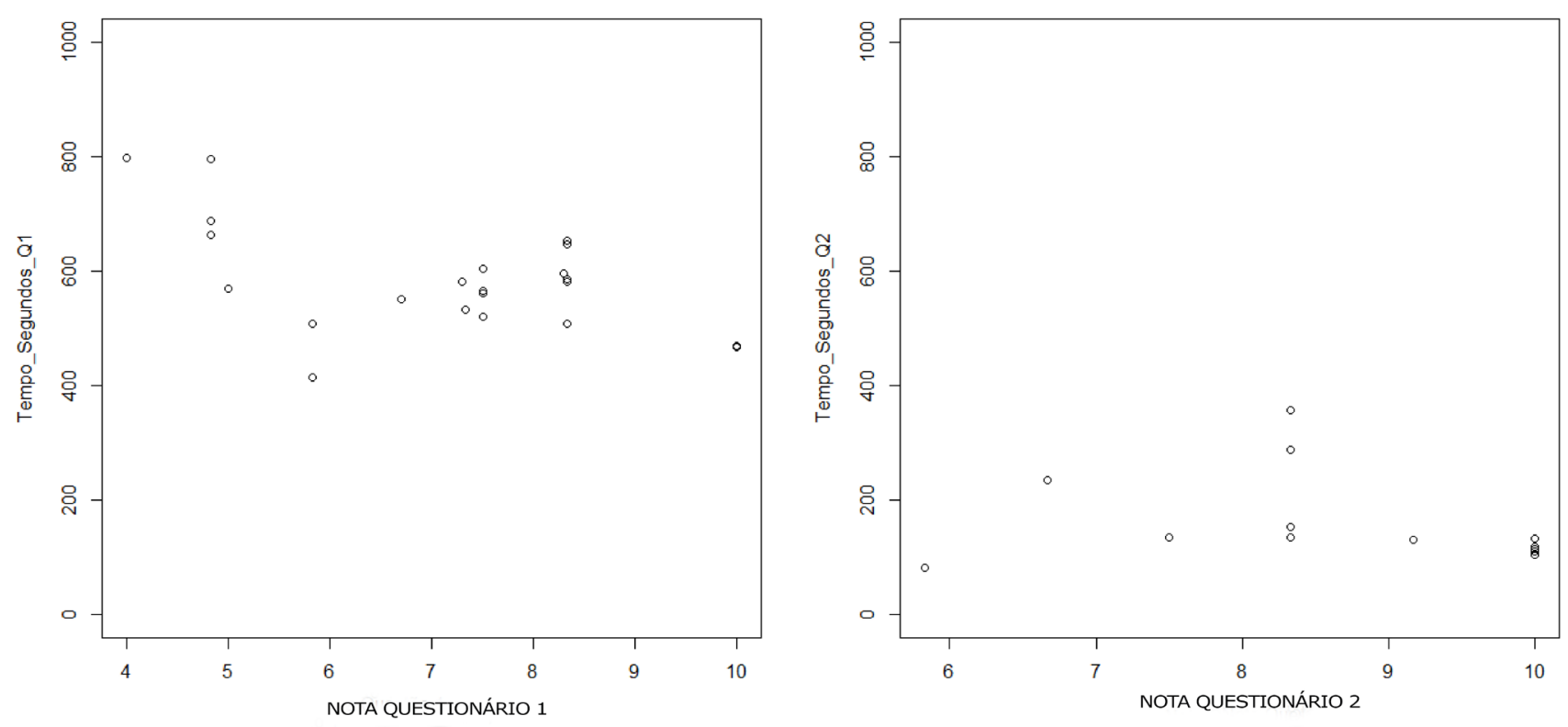

Figura 3 - Comparação do tempo utilizado pelos estudantes para responder o questionário antes e depois da aula. Ribeirão Preto-SP, 2013.

compreensão da importância do gerenciamento de custos em enfermagem, das consequências dos custos das coberturas utilizadas na realização de curativos para o serviço de Enfermagem, do reconhecimento dos fatores que dificultam o gerenciamento de custos de coberturas para curativos nos serviços de Enfermagem e da valorização de informações que contribuem para a adoção de 
soluções para problemas inerentes ao gerenciamento de custos de coberturas para curativos nos serviços de saúde, conforme descrito abaixo:

O foco da equipe de Enfermagem deve estar voltado para a conscientização na prevenção do desperdício de materiais, independentemente do custo e da quantidade destes. Acredito que a melhor maneira para a prevenção do desperdício é a constante capacitação técnica dos profissionais e a educação sobre a questão de custos de uma instituição de saúde (Estudante 3).

Em primeiro lugar, adotar um método para prevenção de LPP, pois assim previnem-se gastos futuros. Depois, orientar os profissionais a utilizarem o material de forma que não haja desperdícios. Utilizar primeiramente um curativo indicado que for de menor valor e, caso não haja melhora da ferida, utilizar outros tipos de curativo (Estudante 4).

Acredito que a equipe deve sempre atender à demanda da população de acordo com a classificação dos estágios da ferida, evitando assim o desperdício de materiais ou uso inadequado, e, com isso, a impossibilidade de cura da lesão. $\mathrm{O}$ prolongamento do paciente na unidade fará com que se aumente a demanda de materiais e o custo para o serviço. Dessa forma, devem-se classificar os materiais a serem utilizados em A, B e C, para que se saiba qual material deverá ser estocado com maior cuidado e controle, racionalizando os gastos. Cabe ressaltar que a capacitação da equipe sobre a racionalização dos materiais sem a perda da qualidade da assistência é muito importante para a melhoria da qualidade do serviço (Estudante 6).

Inicialmente, como os pacientes na enfermaria já se encontram com lesões por pressão, é necessário garantir que a equipe de enfermagem fará os curativos de forma adequada. Para isso, é necessário estabelecer um protocolo do manuseio de feridas e, assim, padronizar o tratamento visando a um cuidado integral do paciente e ao uso racional e adequado das coberturas. Posteriormente, é necessário estabelecer um protocolo na unidade para prevenção de lesões por pressão, e adequá-lo às necessidades de cada paciente (Estudante 10).

A equipe deve trabalhar no sentido de otimizar os recursos disponíveis, evitando dessa forma o desperdício de materiais, medicamentos. É importante também a capacitação dos profissionais de saúde no que se refere ao manuseio de materiais, de modo a que ocorra o mínimo de procedimentos incorretos possível, reduzindo assim os custos e melhorando a qualidade da assistência, promovendo a segurança do paciente (Estudante 15).

Como estratégia, a utilização de coberturas que possam permanecer por mais tempo ajuda na diminuição de custos e na otimização do tempo do profissional, uma vez que o curativo será trocado menos vezes. Porém, além de pensarmos nos custos, devemos pensar no custo-benefício ao cliente, uma vez que será manipulado menos vezes para troca do curativo (Estudante 18).

Primeiramente, devem-se estabelecer metas para a resolução da LPP visando ao risco-benefício do paciente. Deve-se reduzir a troca de curativos, se possível e conforme protocolos, a fim de: causar menor desconforto ao paciente devido à manipulação da ferida; minorar os gastos/ uso de materiais; diminuir o trabalho da equipe de Enfermagem, disponibilizando mais tempo para otimizar a assistência a outros pacientes (Estudante 24).

A melhor forma de reduzir os custos com coberturas para lesão por pressão é a equipe de saúde realizar a prevenção de tais feridas, seguindo protocolo de prevenção de lesão por pressão (Estudante 26).

É necessário que toda a equipe de saúde esteja comprometida com o trabalho e se dedique. Além 
disso, também são importantes a capacitação e a educação constantes dos profissionais, para que eles aprendam a usar os produtos mais adequados e com os custos mais viáveis, sem desperdícios. Outra questão fundamental é a prevenção do aparecimento de feridas, neste caso, de lesões por pressão, por meio de condutas muitas vezes simples, mas que fazem toda a diferença (Estudante 25).

Na análise do questionário referente à avaliação da proposta desenvolvida em sala, à autoavaliação e à avaliação do grupo, realizada por dezoito estudantes, foi atribuída nota média de 96,34 com mediana de 97,73 e desvio padrão de 4,21, evidenciando predominância de desempenho excelente e satisfatório e inexistência de desempenho razoável ou insatisfatório, no atendimento dos requisitos estabelecidos.

Os aspectos avaliados como satisfatórios na primeira parte referem-se às perguntas "Os sites e textos on-line atenderam a suas necessidades de informação em relação ao tema estudado?" e "A apresentação é esteticamente atraente (tipo e tamanho da fonte, cores, tabelas, animações, imagens) e utilizada de forma eficaz na transmissão do conteúdo?", algo realizado por cinco $(27,78 \%)$ estudantes. Na segunda parte, o trabalho em grupo foi considerado satisfatório por quatro $(22,22 \%)$ estudantes. Os demais requisitos foram avaliados como excelentes por no mínimo quinze $(83,33 \%)$ estudantes.

As conclusões apresentadas pelos estudantes evidenciaram: reconhecimento da importância da abordagem da temática de custos na formação do enfermeiro; satisfação com as estratégias de simulação da realidade, ativas e colaborativas, utilizando as tecnologias da informática e informação como ferramentas de apoio ao ensino de graduação; sugestões relacionadas ao tempo disponibilizado para as atividades em aula, conforme descrito abaixo:

Pude vivenciar situações ainda não vistas. O controle de materiais é uma função importante do enfermeiro, por isso ele deve conhecer as necessidades da unidade em que trabalha para evitar gastos indevidos, visando a uma boa utilização dos recursos financeiros, aumentando a qualidade do trabalho e do atendimento ao paciente (Conclusão 4).

A simulação realizada com auxílio do Moodle permite ao estudante desenvolver raciocínio prático e lógico sobre gerenciamento de recursos físicos e materiais. Estimula a aprendizagem do aluno ao proporcionar momentos de reflexão para tomada de decisão na perspectiva de um enfermeiro. Adicionalmente, o aluno poderá entender melhor sobre Sistema de Custeio, Benefício e Custo, avaliação e classificação de feridas, relacionando-os à utilização de cobertura e manejo de materiais adequados, entre outros. Cabe ressaltar que a realização da atividade em grupo permite que os alunos possam tirar suas dúvidas e refletir sobre as mesmas (Conclusão 6).

A aula foi muito produtiva, pois tivemos a oportunidade de simular uma atividade que está sob responsabilidade do enfermeiro, com isso pudemos nos deparar com prováveis dificuldades que encontraremos no nosso ambiente de trabalho. Também notamos como ponto positivo o fato de a aula não ter sido expositiva, pois participamos da atividade de forma ativa, facilitando nosso aprendizado (Conclusão 10).

O modelo de aprendizagem em informática utilizando tecnologias de informação por meio do Moodle proporciona uma interatividade com acesso a vários conteúdos on-line de referências e, assim, proporcionando uma retomada de conhecimento. Deve-se introduzir este modelo de aula em mais disciplinas (Conclusão 12).

Achei muito dinâmica a aula, gostei dessa experiência pois acredito que trouxe a todos muito aprendizado. Mas poderia ter sido em maior tempo ou em maior número de aulas (Conclusão 13).

A aula contribui muito no sentido de nos aproximar de uma realidade ainda não trabalhada: 
"gerenciamento de custos de materiais". Os métodos utilizados, materiais, exercícios nos aproximam da prática a ser desenvolvida neste último período do curso de graduação. Foi muito produtivo, pois permitiu a participação de todos os alunos, bons momentos de discussão e a construção de conhecimentos (Conclusão 15).

A atividade na Tele-enfermagem foi muito produtiva e dinâmica. E nos fez pensar como um enfermeiro, e nos colocou na realidade da nossa prática. Creio que deve permanecer na grade, e é de grande importância (Conclusão 18).

A atividade proporcionou uma primeira aproximação às atribuições do enfermeiro como gestor de materiais, tomando como base o cuidado individual ao paciente. Por meio da leitura e realização da tarefa, pudemos sentir como é desempenhar uma fração da administração da unidade (Conclusão 23).

A atividade utilizando a informática nos possibilita simular a prática do cotidiano do enfermeiro. Prende mais a atenção do aluno, devido à necessidade de tomar decisões para realizar o exercício dentro do tempo de duração da aula. A realização dos exercícios durante a aula nos permite tirar dúvidas na hora. Ainda nos possibilita sermos avaliados pelo docente quanto à presença e participação na aula e capacidade de raciocínio sem a tensão de uma prova (Conclusão 24).

Eu achei muito interessante e dinâmica a aula. Foi bem legal e interativa, possibilitando-nos um bom aproveitamento e excelente aprendizado. Entretanto, acho que o tempo foi muito pouco e insuficiente diante da riqueza e importância desse assunto (Conclusão 25).

A aula proposta foi muito produtiva, pois nos fez retomar conhecimentos e agregar novas perspectivas de forma ativa (Conclusão 26).

A atividade proporcionou uma vivência da prática que encontraremos no campo de trabalho, pois medidas de controle e gerenciamento fazem parte das competências e habilidades do enfermeiro; além disso, o modo como tal exercício foi realizado, em duplas, e as referências disponíveis facilitaram o aprendizado (Conclusão 28).

\section{Conclusão}

Os resultados permitem reconhecer as contribuições da metodologia WebQuest para a abordagem de conteúdos inerentes à temática de gerenciamento de custos de materiais em Enfermagem e a viabilidade de sua aplicação no ensino de graduação, ao adotar a mediação pedagógica enquanto aspecto central no processo ensino-aprendizagem.

Ademais, vale ressaltar a importância da regulação interativa, baseada na premissa de não oferecer respostas prontas aos alunos, mas somente mostrar caminhos ou possibilidades que exigem escolhas a partir de critérios ou argumentos consistentes no cumprimento de tarefas. Tais elementos gradativamente evoluem da característica supletiva para a generativa, constando objetivos e passos claramente explicitados, que simulam o processo de gerenciamento de custos de recursos materiais nos serviços de saúde.

A proposta instrucional encontra respaldo em um projeto institucional para a ampliação do uso de ambientes virtuais de aprendizagem, favorável à adoção e à ampliação do uso da plataforma Moodle, na modalidade presencial. Assim, tal projeto deve ser considerado como exemplo factível, por meio da utilização de recursos tecnológicos simples e acessíveis.

Enquanto experiência iniciada em 2013, a carga horária reduzida é considerada como maior desafio, mas também pode ser entendida como oportunidade para adoção de novas estratégias ou recursos didáticos diferenciados em comparação com as aulas expositivas.

A implementação e a avaliação junto aos alunos são consideradas primordiais para ajustes necessários e até mesmo para subsidiar mudanças futuras, a partir da avaliação do impacto na aprendizagem, a ser ampliado nas turmas subsequentes. 
Finalmente, a principal implicação refere-se ao tempo restrito para implementação junto aos alunos na modalidade totalmente presencial. Como consequência, as tarefas a serem cumpridas e as sugestões de leituras devem ser direcionadas para aspectos fundamentais da temática em formato atraente e convincente para o aluno, enquanto reconhecimento da relevância do tema na formação e atuação profissional do enfermeiro e iniciativa que favorece a ampliação de investimentos futuros na capacitação docente, na modernização das estruturas e recursos existentes, bem como na adoção de estratégias de aprendizagem inovadoras.

\section{Nota}

$1 \mathrm{O}$ termo "lesão por pressão" (LPP) substitui "úlcera por pressão" (UPP) (NPUAP, 2016).

\section{Referências Bibliográficas}

LIMA, Marcio Roberto \& NASCIMENTO, S. S. "Web 2.0: Plataforma para la Reconfiguración de la Educación en la Cibercultura". Alteridad. Revista de Educación, vol. 8, n. 2, 2013, pp. 144-154.

MARUXO, Harriet Bárbara; PRADO, C.; ALMEIDA, D. M., TOBASE, L.; GROSSI, M. G. \& VAZ, D. R. "Webquest e História em Quadrinhos na Formação de Recursos Humanos em Enfermagem". Revista da Escola de Enfermagem da USP, vol. 49, spe, 2015, pp. 68-74.

MAXWELL, Susan M. "WebQuests: A Viable Solution to Meeting the Continuing Professional Education Needs of Home Health Care Nurses". Home Health Care Manag Pract., vol. 21, n. 3, 2009, pp. 171-176.

NATIONAL PRESSURE Ulcer Advisory Panel (NPUAP) Announces a Change in Terminology from Pressure Ulcer to Pressure Injury and Updates the Stages of Pressure Injury. Washington, 13 abr. 2016. Disponível em <http://www.npuap.org/national-pressure-ulcer-advisory-panel-npuap-announces-a-change -in-terminology-from-pressure-ulcer-to-pressure-injury -and-updates-the-stages-of-pressure-injury $>$. Acessado em $1^{\circ}$ jun. 2018.

PEREIRA, Marta Cristiane Alves; MELO, M. R. A. G.; SILVA, A. S. B. \& ÉVORA, Y. D. M.
"Evaluation of a Webquest on the Theme 'Management of Material Resources in Nursing' by Undergraduate Students". Rev Lat Am Enfermagem, vol. 18, n. 6, 2010, pp. 1107-1114. Available from: http://www.scielo.br/ pdf/rlae/v18n6/10.pdf. Access on $1^{\circ}$ may 2018.

; ÉVORA, Y. D. M.; CAMARGO, R. A. A.; TEIXEIRA, G. R. S.; GRUZ, A. G. A. \& GIAVATTA, H. "Ambiente Virtual de Aprendizagem sobre Gerenciamento de Custos de Curativos em Úlceras por Pressão". Rev Eletr Enf., vol. 16, n. 2, 2014, pp. 321-329. Disponível em: <https://www.fen. ufg.br/revista/v16/n2/pdf/v16n2a07.pdP>. Acessado em $1^{\circ}$ maio 2018 .

PIERCY, Emily Carpenter. "Using WebQuests to Promote Active Learning". J Contin Educ Nurs., vol. 35, n. 5, 2004, pp. 200-201.

SANDORD, Julie; TOWNSEND-ROCCHICCIOLI, J.; TRIMM, D. \& JACOBS, M. The Webquest: Constructing Creative Learning. J Continuing Educ Nurs., vol. 41, n. 10, 2010, pp. 473-479.

SILVA, Marco \& CLARO, T. "A Docência Online e a Pedagogia da Transmissão". Rev B. Téc. Senac, vol. 33, n. 2, 2007, pp. 1-12. Disponível em: <http://www. bts.senac.br/index.php/bts/article/view/301/284>. Acessado em $1^{\circ}$ maio 2018.

TEIXEIRA, Carla Regina de Souza; PEREIRA, M. G. A.; KUSUMOTA, L.; GAIOSO, V. P.; MELLO, C. L. \& CARVALHO, E. G. "Avaliação dos Estudantes de Enfermagem sobre a Aprendizagem com a Simulação Clínica". Rev. Bras. Enferm., vol. 68, n. 2, 2015, pp. 311-319. Disponível em: <http://www.scielo. br/pdf/reben/v68n2/0034-7167-reben-68-02-0311.pd† $\gg$. Acessado em $1^{\circ}$ maio 2018.

TIBES, Chris Mayara; DIAS, J. D.; WESTIN, U. M.; DOMINGUES, A. N.; ZEM-MASCARENHAS, S. H. \& ÉVORA, Y. D. M. "Desenvolvimento de Recursos Educacionais Digitais para o Ensino de Enfermagem". Rev Enferm Ufpe On Line., vol. 11, Supl. 3, 2017, pp. 1326-1334. Disponível em: <https:// periodicos.ufpe.br/revistas/revistaenfermagem/article/ view/13972/16815>. Acessado em $1^{\circ}$ maio 2018.

ZHENG, Robert; PEREZ, J.; WILLIAMSON, J. \& FLYGARE, J. "WebQuests as Perceived by Teachers: Implications for Online Teaching and Learning". Journal of Computer Assisted Learning, vol. 24, n. 4, 2008, pp. 295304. Available from: >http://internationalprofessor. com/UNMS/webquest.pd† $\gg$. Access on $1^{\circ}$ may 2018.

Publicado em 12/06/2018. 